\title{
Viscoelastic effects on the jetting-dripping transition in co-flowing capillary jets
}

\author{
J. M. MONTANERO ${ }^{1}$ AND A. M. GAÑ ÁN-CALVO \\ ${ }^{1}$ Departamento de Ingeniería Mecánica, Energética y de los Materiales, \\ Universidad de Extremadura, E-06071 Badajoz, Spain \\ ${ }^{2}$ Departamento de Ingeniería Aeroespacial y Mecánica de Fluidos, \\ Universidad de Sevilla, E-41092 Sevilla, Spain
}

(Received 31 March 2008 and in revised form 2 June 2008)

Linear hydrodynamics stability analysis is used to determine the influence of elasticity on the jetting-dripping transition and on the temporal stability of non-axisymmetric modes in co-flowing capillary jets. The critical Weber number for which axisymmetric perturbations undergo a transition from convective to absolute instability is calculated from the spatio-temporal analysis of the dispersion relation for Oldroyd-B liquids, as a function of the density and viscosity ratios, and the Reynolds and Deborah numbers. Elasticity increases the critical Weber number for all cases analysed and, consequently, fosters the transition from jetting to dripping. The temporal analysis of the dispersion relation for the $m=1$ lateral mode shows that elasticity does not affect its stability.

\section{Introduction}

The controllable production of small flowing particles has been extensively investigated because of its relevance to an immense field of practical and industrial applications, ranging from chemical engineering to bio-industry. Different mechanisms to produce dispersions of drops have been considered (Basaran 2002), including the use of surfactants (Eggleton, Tsai \& Stebe 2001), electrical forces (de la Mora 2007), extensional flows (Taylor 1934; Cohen \& Nagel 2002), thermal gradients, and, more recently, concentrated photon irradiation (Schroll et al. 2007). Appropriate extensional flows can be obtained by a co-flowing outer fluid, which reduces the volume of the issuing drops and results in near monodispersity. Among the extensional flows analysed, flow focusing (Gañan-Calvo 1998) is attractive because of its purely hydrodynamic nature and continuous high-rate production. In the original flow focusing device, a moderately high Reynolds-number liquid jet focused by a gas breaks up into droplets about two orders of magnitude smaller than the source liquid stream. Several alternatives to the original design have been considered over the last few years. In particular, a double flow focusing arrangement has recently been proposed to produce submicrometre liquid jets yielding nanoparticles (GañánCalvo et al. 2007). A coherent understanding of the micro- and nano-scale interfacial flows appearing in all these configurations is essential for a rational design and manufacturing strategy.

When a liquid is injected from a nozzle into a fluid bath or stream, several breakup modes are observed. For low injection velocities, droplets are formed periodically at the nozzle and no jet is observed (dripping). As the flow rate increases, dripping gives 
way to jetting. While dripping produces drops at the nozzle, jetting features a long jet that may extend several orifice diameters downstream and then breaks into droplets through Rayleigh instability. Steady micro-jetting gives rise to a good productivity and well-controlled small drop size. On the other hand, dripping gives rise to much larger droplets under comparable Reynolds and Weber number conditions. Dripping usually yields a highly monodisperse spray, but it may also exhibit bidisperse or polydisperse distributions (Coullet \& Mahadevan 2005). Therefore, it is of interest and importance to understand the mechanisms of instability underlying the jetting-dripping $(\mathrm{J}-\mathrm{D})$ transition, which would allow one to map that transition and determine the jetting conditions yielding the smallest possible jet diameter.

Although the mechanism of jet breakup is highly nonlinear, a linear hydrodynamic stability analysis may, however, be a helpful tool to predict breakup and instability transition occurrence. Among the different oscillation modes with different azimuthal wavenumbers $m$ contained in natural small disturbances of capillary jets, axisymmetric $(m=0)$ modes are generally responsible for instability transitions and breakage processes associated with surface tension (Leib \& Goldstein 1986; Lin 2003). If a jet issues from a stationary source, its behaviour is essentially determined by whether the perturbations are convected downstream for all wavenumbers (convective instability), or some of them can travel upstream while growing (absolute instability). The relationship between these two scenarios (convective and absolute instability) and the jetting and dripping regimes has been already reasonably established (Huerre \& Monkewitz 1990; Lin 2003; Guillot et al. 2007). The calculation of the parameter values for which a basic flow undergoes a convective/absolute (C/A) instability transition can be made analytically tractable at the expense of geometrical simplifications. If the model includes all significant effects (viscosity, inertia, interfacial tension forces, etc.), and the basic flow around which infinitesimal perturbations are considered is realistic, then this approach provides reliable predictions for the J-D transition.

Consider a manifold capillary comprising a liquid stream that envelopes a much thinner inner capillary jet of a second liquid immiscible with the first. Assume that the basic flow in the manifold capillary is characterized by constant velocity and pressure, and can be considered as infinite in terms of the inner jet radius. These conditions are approximately satisfied in several co-flowing configurations (Suryo \& Basaran 2006; Guillot et al. 2007). An example is the flow beyond the orifice of a double flow focusing arrangement (Gañán-Calvo et al. 2007), where the manifold capillary is in turn focused by a third current of a gas forced through a small round orifice. An analysis of the $\mathrm{C} / \mathrm{A}$ instability transition for axisymmetric $(m=0)$ perturbations in the limit of small Reynolds number showed that those perturbations are convected downstream provided that the jet velocity exceeds a certain threshold (Gañán-Calvo et al. 2007). Interestingly, this threshold depends on the viscosities and interfacial tension but is independent of the jet radius (we call this 'unconditional jetting'). This theoretical prediction was confirmed by experiments using a double flow focusing device (Gañán-Calvo et al. 2007).

It is well-known that non-Newtonian liquids can exhibit responses that differ drastically from those of Newtonian liquids in most free-surface flows. Even for small flow rates, the small dimensions of the capillary jets and the very large frequencies arising, for instance, in flow focusing devices cause large strain rates and extremely fast events capable of distorting the microstructure of the liquids (Eggers \& Villermaux 2008), and thus inducing viscoelastic effects. For this reason, increasing attention is being paid to non-Newtonian fluids in microfluidics. Indeed, the study of rheological 
effects is currently a vigorous area of microfluidic research (Stone, Stroock \& Adjari 2004; Eaggers \& Villermaux 2008).

The numerous rheological effects can probably be best elucidated by dissecting, isolating and analysing them separately. In the present paper, we mainly focus our attention on the influence of elasticity on the $\mathrm{J}-\mathrm{D}$ transition appearing in co-flowing jets of Oldroyd-B liquids. In the Oldroyd-B model (Joseph 1990), the elastic effects increase with the Deborah number, which is defined as the ratio of the stress relaxation time to the characteristic time. The characteristic time takes very small values for micrometre and submicrometre jets produced by flow focusing or microfluidic devices, and thus one obtains large values of the Deborah number even for small stress relaxation times. Therefore, one may expect a significant influence of elasticity on the $\mathrm{J}-\mathrm{D}$ transition in flow focusing and microdevices operating with viscoelastic liquids.

Temporal linearized stability analysis for axisymmetric disturbances shows that an Oldroyd-B jet exhibits a faster growth rate than a Newtonian one with the same Ohnesorge number, indicating that non-Newtonian jets are more unstable than their Newtonian counterparts (Brenn, Liu \& Durst 2000; Funada \& Joseph 2003). The most unstable wavelength on a viscoelastic jet is shorter than on a comparable Newtonian one, which implies that smaller drops would form for viscoelastic liquids. This theoretical result has been confirmed by simulations (Zhou, Yue \& Feng 2006). The effect of a small elasticity on non-axisymmetric modes evolving in a single column (Bauer 1986) and in a jet moving in an inviscid gaseous environment (Liu \& Liu 2006) has also been examined. The results indicated that elasticity does not modify the stability of these latter modes though, and only slightly affects their frequencies and damping factors.

The $\mathrm{J}-\mathrm{D}$ transition is controlled by the ratio of the time scale required for pinch-off to a convective time scale. For large flow velocities, the convective time scale is smaller than that required for pinch-off, transport dominates, and a jet forms. The fact that viscoelastic properties accelerate the pinch-off in the linear regime (Brenn et al. 2000; Funada \& Joseph 2003) suggests that those properties make jetting take place at jet velocities larger than in the Newtonian case (which might be the case in (Anna \& Mayer 2006). A spatiotemporal analysis is necessary to confirm that expectation, and to determine the critical values of the Weber number for which the $\mathrm{J}-\mathrm{D}$ transition occurs.

In the present paper, we describe a linear spatiotemporal stability analysis for axisymmetric $(m=0)$ perturbations in a co-flowing system of two viscoelastic liquids moving with the same velocity to determine the parametric values for which a $\mathrm{C} / \mathrm{A}(\mathrm{J}-$ D) instability transition takes place. Our study extends the previous one by Funada \& Joseph (2003) in two directions: a spatiotemporal analysis is carried out, and elastic effects on both the inner jet and the co-flowing liquid are considered. In addition, we evaluate the influence of elasticity on the damping factor characterizing the evolution of the lateral $(m=1)$ mode.

\section{Linear stability}

Consider a cylindrical jet of radius $R$ moving in the axial direction into another liquid co-flowing with the same velocity $V$ (see e.g. Gañán-Calvo et al. 2007). The flow configuration considered is sketched in figure 1. The perturbed shape of the jet, velocity, and pressure may be expressed as $r=f(\theta, z, t), \boldsymbol{v}(\boldsymbol{r}, t) \equiv[u(\boldsymbol{r}, t), v(\boldsymbol{r}, t), w(\boldsymbol{r}, t)]$, and $p(\boldsymbol{r}, t)$, respectively. In what follows, we shall make all the variables dimensionless using the inner jet radius $R$, velocity $V$, time $R / V$, and momentum $\rho_{1} V^{2}$ as the 


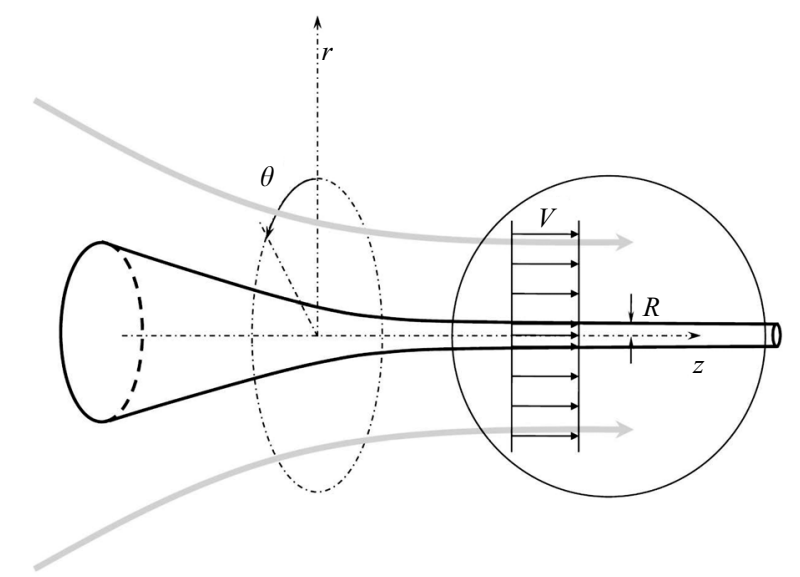

FIGURE 1. Sketch of the flow configuration considered. The circled region is where the analysis presented here applies.

characteristic length, velocity, time, and pressure, respectively, where $\rho_{1}$ is the inner liquid density. Using a Lagrangian frame of reference moving with both fluids, we propose the following dependence for the hydrodynamic fields and the interface position:

$$
\begin{gathered}
\boldsymbol{v}_{j}(\boldsymbol{r}, t)=\varepsilon\left\{U_{j}(r), V_{j}(r), W_{j}(r)\right\} \mathrm{e}^{\mathrm{i}(m \theta+k z-\omega t)}+\text { c.c. }, \\
p_{j}(\boldsymbol{r}, t)-\frac{\delta_{j 1}}{W e}=\varepsilon P_{j}(r) \mathrm{e}^{\mathrm{i}(m \theta+k z-\omega t)}+\text { c.c. } \\
f(\theta, z, t)-1=\varepsilon F \mathrm{e}^{\mathrm{i}(m \theta+k z-\omega t)}+\text { c.c. }
\end{gathered}
$$

where $j=1$ and 2 stand for the inner jet and co-flowing liquid, respectively, $m$ is the azimuthal wavenumber, $k=k_{r}+\mathrm{i} k_{i}$ the axial wavenumber, $\omega=\omega_{r}+\mathrm{i} \omega_{i}$ the frequency, $W e=\rho_{1} V^{2} R / \sigma$ the Weber number, $\sigma$ the interfacial tension, and $\delta_{i j}$ the Kronecker delta.

The rheological behaviour of a viscoelastic material can be described by the Oldroyd-B model (Joseph 1990):

$$
(1+\tau \mathrm{G}) \sigma_{i k}=2 \mu_{0}(1+\lambda \mathrm{G}) D_{i k},
$$

where $\mathrm{G}[\mathrm{A}] \equiv \mathrm{d} A / \mathrm{d} t-w A+A w$ is the upper convected derivative operator, $\mathrm{d} / \mathrm{d} t$ is the material derivative, $w$ is the antisymmetric part of $D, \sigma_{i k}$ is the (non-equilibrium) stress tensor, $\mu_{0}$ the Newtonian viscosity, and $D_{i k}$ the strain rate tensor. The model also involves the two rheological parameters $\tau$ and $\lambda$. For small perturbations around our motionless (in the Lagrangian frame of reference) basic flow, the upper convected derivative operator $\mathrm{G}$ can be approximated by the partial derivative $\partial / \partial t$, and thus the Oldroyd-B model yields

$$
\sigma_{i k}+\tau \frac{\partial \sigma_{i k}}{\partial t}=2 \mu_{0}\left(D_{i k}+\lambda \frac{\partial D_{i k}}{\partial t}\right),
$$

where $\tau$ and $\lambda$ are the (dimensionless) stress and strain relaxation times. These parameters are also known as the Deborah number and the (dimensionless) retardation time, respectively. For $\lambda=0,(2.3)$ reduces to the Maxwell model, widely used to describe a viscoelastic material in the linear response regime. If one consistently assumes a temporal dependence of the form $\mathrm{e}^{-\mathrm{i} \omega t}$ for $\sigma_{i k}$ and $D_{i k}$ to the order $\varepsilon$, then 
the constitutive relation (2.3) to that order is

$$
\sigma_{i k}=2 \mu_{0} \frac{1-\mathrm{i} \lambda \omega}{1-\mathrm{i} \tau \omega} D_{i k} .
$$

The parametric values for which the system becomes temporally unstable are obtained from the dispersion relation by imposing the condition of zero growth rate $\left(\omega_{i}=0\right)$, and thus the critical motion corresponds to an harmonic oscillation of frequency $\omega_{r}$. If the oscillation period is large in terms of $\tau$ and $\lambda\left(\left|\omega_{r}\right| \tau,\left|\omega_{r}\right| \lambda \ll 1\right)$, the liquid can be regarded as Newtonian. On the other hand, if the oscillation period is of the same order as or even larger than the stress relaxation time $\left(\left|\omega_{r}\right| \tau,\left|\omega_{r}\right| \lambda \sim 1\right.$ or $\left.\left|\omega_{r}\right| \tau,\left|\omega_{r}\right| \lambda>1\right)$, the elastic effects are significant and may affect the $\mathrm{J}-\mathrm{D}$ transition. It must be noted that large values of $\left|\omega_{r}\right|$ lead to large values of the strain rate $D_{i k}$ and, consequently, may yield a failure of the linear model (2.4).

If one introduces (2.1) and (2.4) into the equations of conservation of mass and momentum, and neglects terms in $\varepsilon^{2}$, one obtains

$$
\begin{gathered}
U_{j}^{\prime}+U_{j} / r+\mathrm{i} m V_{j} / r+\mathrm{i} k W_{j}=0, \\
-\rho^{\delta_{j 2}} \mathrm{i} \omega U_{j}+P_{j}^{\prime}=\frac{\mu^{\delta_{j 2}}}{R e} \frac{1-\mathrm{i} \lambda_{j} \omega}{1-\mathrm{i} \tau_{j} \omega}\left[U_{j}^{\prime \prime}+U_{j}^{\prime} / r-\left(m^{2}+1\right) U_{j} / r^{2}-k^{2} U_{j}-2 \mathrm{i} m V_{j} / r^{2}\right], \\
-\rho^{\delta_{j 2}} \mathrm{i} \omega V_{j}+\mathrm{i} m P_{j} / r=\frac{\mu^{\delta_{22}}}{\operatorname{Re}} \frac{1-\mathrm{i} \lambda_{j} \omega}{1-\mathrm{i} \tau_{j} \omega}\left[V_{j}^{\prime \prime}+V_{j}^{\prime} / r-\left(m^{2}+1\right) V_{j} / r^{2}-k^{2} V_{j}+2 \mathrm{i} m U_{j} / r^{2}\right], \\
-\rho^{\delta_{j 2}} \mathrm{i} \omega W_{j}+\mathrm{i} k P_{j}=\frac{\mu^{\delta_{j 2}}}{\operatorname{Re}} \frac{1-\mathrm{i} \lambda_{j} \omega}{1-\mathrm{i} \tau_{j} \omega}\left(W_{j}^{\prime \prime}+W_{j}^{\prime} / r-m^{2} W_{j} / r^{2}-k^{2} W_{j}\right),
\end{gathered}
$$

where $\rho \equiv \rho_{2} / \rho_{1}$ and $\mu \equiv \mu_{2} / \mu_{1}$ are the ratios between the density $\rho_{2}$ and Newtonian viscosity $\mu_{2}$ of the co-flowing fluid and the corresponding values $\rho_{1}$ and $\mu_{1}$ of the inner jet, and $R e=\rho_{1} V R / \mu_{1}$ is the Reynolds number. The general solution to (2.5), satisfying the regularity conditions

$$
\begin{gathered}
U_{1}(0)=V_{1}(0)=W_{1}^{\prime}(0)=0 \text { for } m=0, \quad U_{1}(0)+\mathrm{i} V_{1}(0)=W_{1}(0)=0 \text { for } m=1, \\
U_{1}(0)=V_{1}(0)=W_{1}(0)=0 \text { for } m \geqslant 2, \quad U_{2}=V_{2}=W_{2}=P_{2}=\text { bounded at } r \rightarrow \infty
\end{gathered}
$$

can be written as

$$
\begin{gathered}
U_{j}(r)=\mathrm{i} c_{j 1} \Theta_{m}^{\prime}(k r)+\mathrm{i} c_{j 2} \Theta_{m}^{\prime}\left(k_{j} r\right)+\mathrm{i} m c_{j 3} \frac{\Theta_{m}\left(k_{j} r\right)}{k_{j} r}, \\
V_{j}(r)=-m c_{j 1} \frac{\Theta_{m}(k r)}{k r}-m c_{j 2} \frac{\Theta_{m}\left(k_{j} r\right)}{k_{j} r}-c_{j 3} \Theta_{m}^{\prime}\left(k_{j} r\right), \\
W_{j}(r)=-c_{j 1} \Theta_{m}(k r)-c_{j 2} k_{j} \frac{\Theta_{m}\left(k_{j} r\right)}{k}, \\
P_{j}(r)=-\frac{\rho^{\delta_{j 2}} \omega c_{j 1}}{k} \Theta_{m}(k r) .
\end{gathered}
$$

Here, $\Theta_{m}$ is the modified Bessel function of the first kind $I_{m}$ if $j=1$, and of the second kind $K_{m}$ if $j=2, k_{1}= \pm\left[k^{2}-\mathrm{i} \omega \operatorname{Re}\left(1-\mathrm{i} \tau_{1} \omega\right) /\left(1-\mathrm{i} \lambda_{1} \omega\right)\right]^{1 / 2}, k_{2}=\left[k^{2}-\mathrm{i} \omega R e \rho / \mu\right.$ $\left.\left(1-\mathrm{i} \tau_{2} \omega\right) /\left(1-\mathrm{i} \lambda_{2} \omega\right)\right]^{1 / 2}$, and $\left\{c_{j 1}, c_{j 2}, c_{j 3}\right\}$ are six arbitrary constants. Note that $(2.7)$ 
is symmetric with respect to $k_{1}$, while the real part of $k$ must be positive to satisfy $(2.6 b)$.

The non-slip condition $\left\{U_{1}=U_{2}, V_{1}=V_{2}, W_{1}=W_{2}\right\}$ at the interface $r=1$ yields a linear system of equations that allows one to obtain $\left\{c_{21}, c_{22}, c_{23}\right\}$ in terms of $\left\{c_{11}, c_{12}, c_{13}\right\}$. The condition of dynamical equilibrium at the interface $r=1$ for the normal and two tangential components leads to

$$
\begin{gathered}
P_{1}-P_{2}+\frac{\mathrm{i}\left(1-m^{2}-k^{2}\right)}{W e \omega} U_{1}=\frac{2}{R e}\left(\frac{1-\mathrm{i} \lambda_{1} \omega}{1-\mathrm{i} \tau_{1} \omega} U_{1}^{\prime}-\mu \frac{1-\mathrm{i} \lambda_{2} \omega}{1-\mathrm{i} \tau_{2} \omega} U_{2}^{\prime}\right), \\
\frac{1-\mathrm{i} \lambda_{1} \omega}{1-\mathrm{i} \tau_{1} \omega}\left(W_{1}^{\prime}+\mathrm{i} k U_{1}\right)=\mu \frac{1-\mathrm{i} \lambda_{2} \omega}{1-\mathrm{i} \tau_{2} \omega}\left(W_{2}^{\prime}+\mathrm{i} k U_{2}\right), \\
\frac{1-\mathrm{i} \lambda_{1} \omega}{1-\mathrm{i} \tau_{1} \omega}\left(\mathrm{i} m U_{1}+V_{1}^{\prime}-V_{1}\right)=\mu \frac{1-\mathrm{i} \lambda_{2} \omega}{1-\mathrm{i} \tau_{2} \omega}\left(\mathrm{i} m U_{2}+V_{2}^{\prime}-V_{2}\right),
\end{gathered}
$$

where use has been made of the kinematic compatibility condition $F=\mathrm{i} U_{j}(1) / \omega$ to eliminate $F$ from (2.8a). The set of equations (2.8) constitutes a linear system of equations for $\left\{c_{11}, c_{12}, c_{13}\right\}$. The solvability condition $\operatorname{Det}\left(\Delta_{i j}\right)=0$, with $\Delta_{i j}$ being the $3 \times 3$ matrix associated with that system of equations, yields the dispersion relation

$$
S_{m}\left(k, \omega, \rho, \mu, R e, W e, \tau_{1}, \tau_{2}, \lambda_{1}, \lambda_{2}\right)=0 .
$$

A practical explicit expression of (2.9) cannot be provided here. A Mathematica notebook containing the dispersion relation can be obtained upon request from the authors.

The most important conclusion drawn from the temporal analysis presented in Montanero \& Gañán-Calvo (2008) for Newtonian liquids is that non-axisymmetric $(m \neq 0)$ perturbations possess negative growth factors over the whole parameter space. Therefore, a transition from convective (jetting) to absolute instability for $m \neq 0$ is not possible for the basic flow analysed. A natural question is whether elasticity could modify the above result. To answer this question, we here perform a temporal analysis ( $k$ real) of (2.9) for the lateral mode $m=1$. For this purpose, we re-write (2.9) in the form

$$
Q_{m}\left(k, \widetilde{\omega}, \rho, \mu, O h, \tau_{1}, \tau_{2}, \lambda_{1}, \lambda_{2}\right)=0,
$$

where $\widetilde{\omega}=\widetilde{\omega}_{r}+\mathrm{i} \widetilde{\omega}_{i} \equiv W e^{1 / 2} \omega$ and $O h \equiv W e^{1 / 2} / R e$ is the Ohnesorge number. This is equivalent to choosing the capillary velocity $V_{c} \equiv\left(\sigma / \rho_{1} R\right)^{1 / 2}$ instead of $V$ as the characteristic velocity, an appropriate choice when the flow is described in our Lagrangian frame of reference. It must be noted that this simplification is exclusive to the basic flow considered here, where both the inner jet and the co-flowing liquid move with the same velocity $V$.

Solutions to $(2.10)$ in the complex plane $\left(\widetilde{\omega}_{r}, \widetilde{\omega}_{i}\right)$ were found numerically for $0<k<1$ and fixed values of the rest of the parameters. Because we were mainly interested in finding possible transitions from stable $\left(\widetilde{\omega}_{i}<0\right)$ to unstable $\left(\widetilde{\omega}_{i}>0\right)$ perturbations, (2.10) was carefully explored close to the $\widetilde{\omega}_{i}=0$ axis, while less attention was paid to other regions of the $\left(\widetilde{\omega}_{r}, \widetilde{\omega}_{i}\right)$-plane. The roots of (2.10) were found by means of the Newton-Raphson method, using as initial guess the value obtained from a linear extrapolation of the two previous solutions. We considered as solutions those satisfying the condition $\left|Q_{1}\right|<10^{-10}$.

In this paper, we mainly focus attention on the $\mathrm{J}-\mathrm{D}$ transition in the co-flowing jets, explained in terms of the C/A instability transition for the axisymmetric mode 
$(m=0)$. In this case, an explicit expression for the dispersion relation (2.9) can be provided:

$$
\begin{gathered}
S_{0} \equiv \mathrm{i} \omega\left[\frac{N\left(k, k_{1}, k_{2}, \mu^{*}\right)}{M\left(k, k_{1}, k_{2}, \mu^{*}\right)}+2\left(1-\mu^{*}\right)\right]-\frac{R e^{*}}{W e}\left(1-k^{2}\right)=0 \\
N \equiv 2 k \mu^{*} k_{1} k_{2}\left[K_{0}\left(k_{2}\right) I_{1}\left(k_{1}\right) k_{1}+I_{0}\left(k_{1}\right) K_{1}\left(k_{2}\right) k_{2}\right]+k\left[k^{2}\left(\mu^{*}-1\right)-k_{1}^{2}+\mu^{*} k_{2}^{2}\right]^{2} \\
\times I_{0}(k) I_{1}\left(k_{1}\right) K_{0}(k) K_{1}\left(k_{2}\right)+4 k^{3} k_{1} k_{2}\left(\mu^{*}-1\right)^{2} I_{0}\left(k_{1}\right) I_{1}(k) K_{0}\left(k_{2}\right) K_{1}(k) \\
-k_{2} I_{1}\left(k_{1}\right) K_{0}\left(k_{2}\right)\left\{\left[k^{4}+k_{1}^{2} k_{2}^{2}+k^{2}\left(k_{1}^{2}-k_{2}^{2}\right)\right] \mu^{*} I_{1}(k) K_{0}(k)\right. \\
\left.+\left[k_{1}^{4}+k^{4}\left(1-2 \mu^{*}\right)^{2}-2 k^{2} k_{1}^{2}\left(\mu^{*}-1\right)\right] I_{0}(k) K_{1}(k)\right\} \\
-k_{1} I_{0}\left(k_{1}\right) K_{1}\left(k_{2}\right)\left\{\left[k^{4}\left(\mu^{*}-2\right)^{2}+2 k^{2} k_{2}^{2} \mu^{*}\left(\mu^{*}-1\right)+\mu^{* 2} k_{2}^{4}\right] I_{1}(k) K_{0}(k)\right. \\
\left.+\left[k^{2}\left(k^{2}-k_{1}^{2}\right)+k_{2}^{2}\left(k^{2}+k_{1}^{2}\right)\right] \mu^{*} I_{0}(k) K_{1}(k)\right\} \\
\quad M \equiv k\left\{\left[k_{2} K_{0}\left(k_{2}\right) K_{1}(k)-k K_{0}(k) K_{1}\left(k_{2}\right)\right]\left(k_{1}^{2}-k^{2}\right) I_{1}(k) I_{1}\left(k_{1}\right)\right. \\
\left.\quad+\mu^{*}\left[k_{1} I_{0}\left(k_{1}\right) I_{1}(k)-k I_{0}(k) I_{1}\left(k_{1}\right)\right]\left(k_{2}^{2}-k^{2}\right) K_{1}(k) K_{1}\left(k_{2}\right)\right\}
\end{gathered}
$$

In the above expressions, $k_{1}= \pm \sqrt{k^{2}-\mathrm{i} \omega R e^{*}}, \quad k_{2}=\sqrt{k^{2}-\mathrm{i} \omega R e^{*} \rho / \mu^{*}}, \quad R e^{*} \equiv$ $\operatorname{Re}\left(1-\mathrm{i} \tau_{1} \omega\right) /\left(1-\mathrm{i} \lambda_{1} \omega\right)$, and $\mu^{*}=\mu\left(1-\mathrm{i} \tau_{1} \omega\right) /\left(1-\mathrm{i} \tau_{2} \omega\right)\left(1-\mathrm{i} \lambda_{2} \omega\right) /\left(1-\mathrm{i} \lambda_{1} \omega\right)$.

To determine the parameter values for which the basic flow undergoes a $\mathrm{C} / \mathrm{A}$ instability transition, one should explore the response of the system to perturbations characterized by a complex axial wavenumber $k$ (spatio-temporal analysis), observed by a fixed observer anchored at the nozzle (Huerre \& Monkewitz 1990). To change the frame of reference from a travelling observer to a fixed one, we just need to replace the wave frequency $\omega$ by $\omega^{\prime}-k$ in the dispersion relation (2.11) (Leib \& Goldstein 1986).

A localized initial distortion spreads in the fluid domain bounded by two fronts moving with velocities $v_{+}^{*}$ and $v_{-}^{*}$. If the distortion is the superposition of perturbations proportional to $\mathrm{e}^{\mathrm{i}\left(k z-\omega^{\prime} t\right)}=\mathrm{e}^{k_{\mathrm{i}}(v t-z)} \mathrm{e}^{\mathrm{i}\left(k_{r} z-\omega^{\prime} t\right)}$, then $v_{ \pm}^{*}$ are calculated as the extremal values of the envelope velocity $v=\omega^{\prime}{ }_{i} / k_{i}$ of those perturbations (van Saarloos 1987, 2003; Guillot et al. 2007). The C/A instability transition occurs for parameter points for which the velocity $v_{-}^{*}$ of the back front vanishes. As demonstrated in the Appendix, this criterion is equivalent to the so-called saddle-point rule, which establishes that the $\mathrm{C} / \mathrm{A}$ instability transition occurs when there is at least one solution of the dispersion relation satisfying $\mathrm{d} \omega^{\prime} / \mathrm{d} k=0$ (zero group velocity) with $\omega_{i}^{\prime}=0$ (Briggs 1964; Huerre \& Monkewitz 1990). Once the parameters $\left\{\rho, \mu, R e, \tau_{1}, \tau_{2}, \lambda_{1}, \lambda_{2}\right\}$ are set, one has to find the value of the Weber number for which $v_{-}^{*}=0$ or, equivalently, $\mathrm{d} \omega^{\prime} / \mathrm{d} k=\omega_{i}^{\prime}=0$. The latter condition can be formulated in terms of four coupled transcendental equations $F_{i}\left(k_{r}, k_{i}, \omega_{r}^{\prime}, W e\right)=0(i=1,2,3$, and 4$)$ for the set of variables $\left\{k_{r}, k_{i}, \omega_{r}^{\prime}, W e\right\}$. In this work, the roots of that system of equations were found numerically by the secant method within the variable space volume delimited by the previous solution and that obtained from a linear extrapolation of the two previous ones. We considered as solutions those satisfying the condition $\left|F_{i}\right|<10^{-10}(i=1,2,3$, and 4).

\section{Results and conclusions}

Viscoelastic liquids in co-flowing devices usually have similar densities $(\rho \simeq 1)$ and retardation times at least one order of magnitude less than the relaxation times $\left(\lambda_{j} \ll \tau_{j}\right)$ (see e.g. Brenn et al. 2000 or Funada \& Joseph 2003 and references therein). 

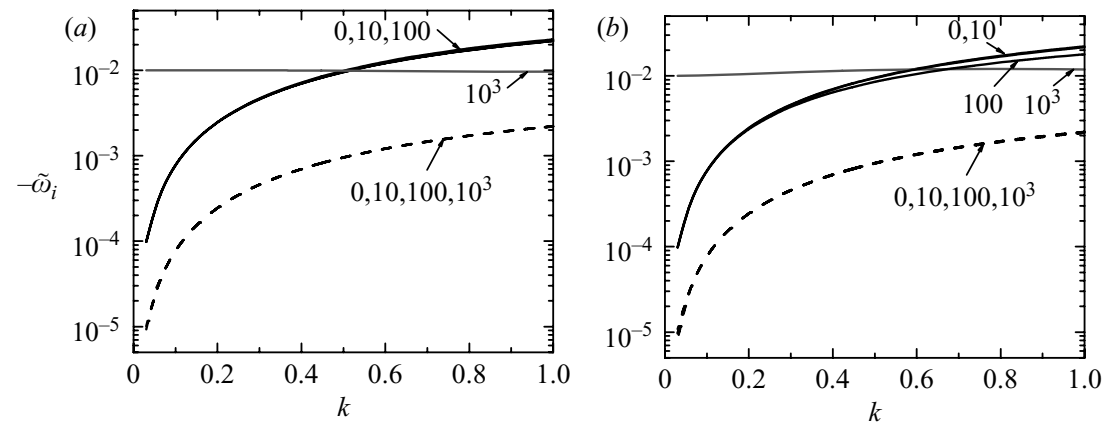

FIGURE 2. Growth factor $\widetilde{\omega}_{i}$ as a function of $k$ for $\mu=1$ and for $O h=10$ (solid lines) and $O h=100$ (dashed lines): $(a) \tau_{2}=0$ and different values of $\tau_{1},(b) \tau_{1}=0$ and different values of $\tau_{2}$, as indicated by the labels. The black curves correspond to overdamped motions, while the grey lines labelled $10^{3}$ correspond to oscillatory motions.

Because of the high dimension of the parameter space, we explored it partially by considering the cases $\rho=1$ and $\lambda_{j}=0$ (Maxwell model). The temporal analysis of (2.10) for the lateral mode $m=1$ is presented in $\S 3.1$, while in $\S 3.2$ we show the spatiotemporal analysis of (2.11) for the axisymmetric mode $m=0$.

\subsection{Temporal analysis of the lateral mode}

For non-surrounded jets $(\rho=\mu=0)$, it is well-known that axisymmetric $(m=0)$ perturbations are stable for $k>1$ and unstable for $0<k<1$. For this reason, in the present study we calculate the growth factors characterizing the dominant lateral motions of co-flowing jets within the interval $0<k<1$. The values of the Ohnesorge number considered correspond to high-Newtonian-viscosity liquids, as are usually employed in experiments. The most important conclusion drawn from the results shown in figure 2 (and others not presented here) is that lateral perturbations possess negative growth factors within the region of parameter space explored, and hence elasticity does not modify their stability. For $O h=100$, the influence of elasticity is negligible even for large values of the Deborah number. The damping factor slightly decreases as $\tau_{j}$ increases for $O h=10$. A transition from overdamped to oscillatory motion takes place between $\tau_{j}=100$ and $10^{3}$ for that value of the Ohnesorge number.

\subsection{Spatio-temporal analysis of the axisymmetric mode}

The main aim of the present work was to determine the influence of elasticity on the $\mathrm{J}-\mathrm{D}$ transition in a capillary flow consisting of two co-flowing liquid jets moving with the same velocity. For this purpose, the critical Weber number for which the C/A instability transition occurs is obtained as a function of the other parameters. When working with millimetric jets, the Deborah number usually takes values less than unity. Figure 3 shows the ratio between the critical Weber number We for $0 \leqslant \tau_{j} \leqslant 1$ and its value $W e_{0}$ for the Newtonian case $\left(\tau_{1}=\tau_{2}=0\right)$. Elasticity slightly increases the critical Weber number. Similar results are obtained when the viscoelastic material is the inner (solid lines) or outer (dashed lines) jet. The effect of elasticity increases as the Reynolds number decreases.

For micrometre and submicrometre jets, the Deborah number may take large values even for small stress relaxation times. Figure 4 shows the J-D transition curves for $\tau_{j}=0,10,10^{2}, 10^{3}$, and $10^{4}$, and different values of the Newtonian viscosity ratio $\mu$. The main result is that elasticity increases the critical Weber number significantly 


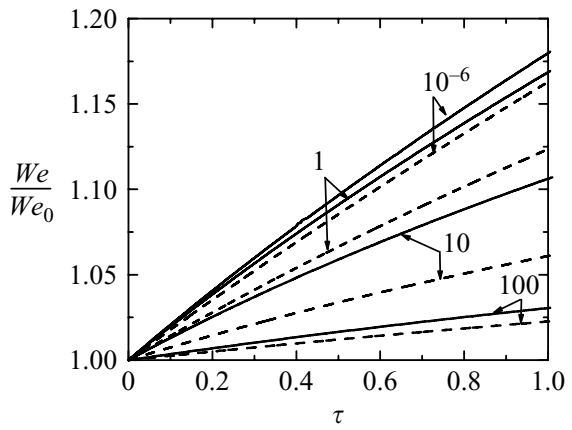

FiguRE 3. Ratio between the critical Weber number $W e$ and its value $W e_{0}$ for the Newtonian case $\left(\tau_{1}=\tau_{2}=0\right)$ both calculated for $\mu=1$. The solid (dashed) lines indicate the dependence on $\tau_{1}\left(\tau_{2}\right)$ for $\tau_{2}=0\left(\tau_{1}=0\right)$. The labels are the values of the Reynolds number.

(a)
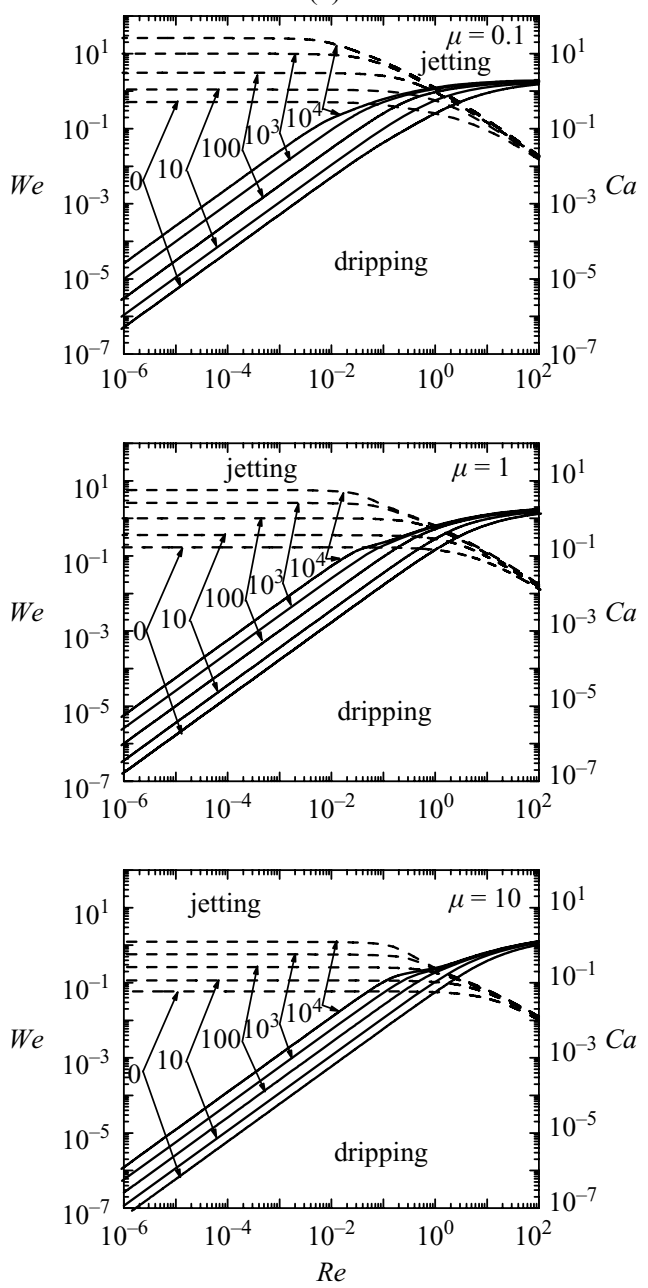

(b)
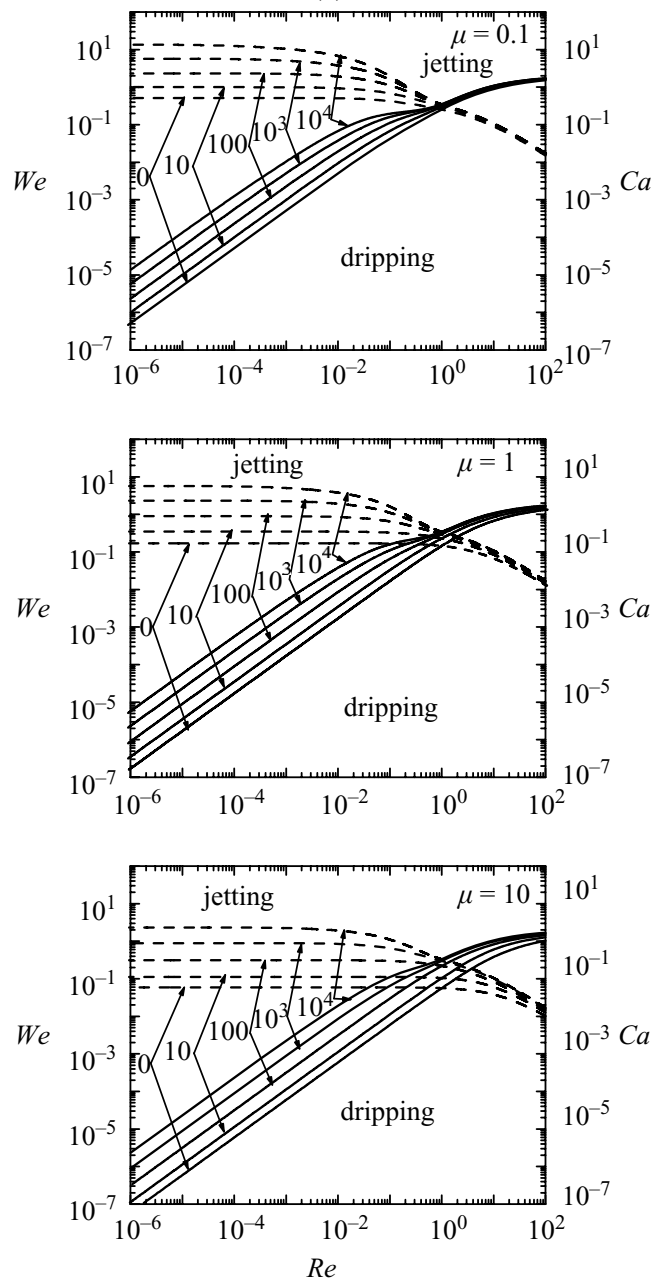

FIGURE 4. Critical value of the Weber (solid lines) and capillary (dashed lines) numbers as a function of the Reynolds number for $\mu=0.1,1$, and 10: $(a)$ for $\tau_{2}=0$ and different values of $\tau_{1},(b) \tau_{1}=0$ and different values of $\tau_{2}$, as indicated by the labels. 

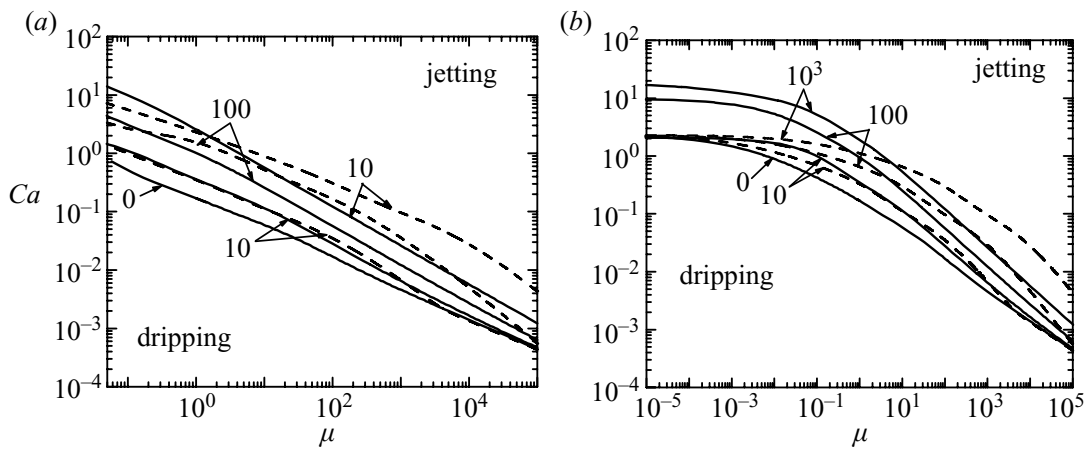

FIGURE 5. Critical value of the capillary number as a function of the viscosity ratio for $(a)$ $R e=10^{-4}$ and $(b) 0.1$. The solid (dashed) lines are the results calculated for $\tau_{2}=0\left(\tau_{1}=0\right)$ and different values of $\tau_{1}\left(\tau_{2}\right)$ as indicated by the labels.

(solid lines) for small Re, which is the most relevant case experimentally. Similar trends are observed for all viscosity ratios analysed. In addition, elasticity plays a similar role in both the inner and outer jet. The dashed lines correspond to the critical values of the capillary number $C a \equiv W e / R e=\mu_{1} V / \sigma$. In the limit $R e \rightarrow 0$, the transition from dripping to jetting takes place in a Newtonian system if and only if $\mathrm{Ca}$ exceeds a critical value, independently of the jet radius (unconditional jetting) (GañánCalvo et al. 2007). Elasticity does not modify this feature, although it increases the critical value. This can also be observed in figure 5, where we show the dependence of the critical capillary number on the viscosity ratio for small-Reynolds-number flows (submicrometre or nanoscale), in particular for $R e=10^{-4}$ and 0.1. For $R e=10^{-4}$, we only present results for $\beta>0.05$ because our algorithm did not satisfactorily converge to the solution sought in the limit $R e \rightarrow 0$ and $\mu \rightarrow 0$ (creeping flow equations are required to properly address this limit case).

To summarize, one can assert that elasticity encourages the instability of infinitesimal perturbations in capillary co-flowing jets moving with the same velocity. It increases the growth rate of the temporally unstable axisymmetric mode (Brenn et al. 2000; Funada \& Joseph 2003), decreases the damping rate of the temporally stable lateral mode (figure 2), and favours the transition from the convective (jetting) to absolute (dripping) instability of the spatiotemporal axisymmetric mode (figures 3 and 4). Note that the latter effect does not imply that the use of viscoelastic liquids necessarily yields the production of drops larger than their Newtonian counterparts. Indeed, the size of the drops is the result of a highly nonlinear breakup process not considered in the present analysis. With increasing deformation, non-Newtonian liquid behaviour leads to a retardation of breakup and to the formation of a structure of drops connected by thin filaments (Eggers \& Villermaux 2008), which may end in drops smaller than their Newtonian counterparts for the same Weber number.

Partial support from the MCYT (Spain) through Grant No. DPI2007-63559 is gratefully acknowledged.

\section{Appendix}

The criterion for determining the $\mathrm{C} / \mathrm{A}$ instability transition originally proposed by van Saarloos $(1987,2003)$ and recently used by Guillot et al. (2007) is based on the analysis of the propagation front velocity, which is found to be very intuitive 
and immediately understandable in physical terms. This is an alternative to the socalled saddle-point criterion proposed by Briggs (1964) and used by most workers in capillary flows, and capillary jets in particular. Although van Saarloos's criterion may be preferable for capillary jets, it has been used without proving its equivalence to the saddle-point criterion. We prove below the equivalence of the two criteria.

Consider a quasi-one-dimensional dispersive system whose dynamical response is the combination of small perturbations proportional to $\mathrm{e}^{\mathrm{i}(k z-\omega t)}=\mathrm{e}^{k_{\mathrm{i}}(v t-z)} \mathrm{e}^{\mathrm{i}\left(k_{r} z-\omega_{r} t\right)}$, with $k=k_{r}+\mathrm{i} k_{\mathrm{i}}$ and $\omega=\omega_{r}+\mathrm{i} \omega_{\mathrm{i}}$ related by a dispersion relation $\omega(k)$. A localized initial distortion spreads in the fluid domain bounded by two fronts moving with velocities $v_{+}^{*}$ and $v_{-}^{*}$ calculated as the extremal values of the envelope velocity $v=\omega_{i} / k_{i}$ of the small perturbations, i.e. $v_{-}^{*}=\omega_{i}^{*} / k_{i}^{*}$ where $\left(\omega^{*}, k^{*}\right)$ is a solution of the dispersion relation for which $\partial\left(\omega_{i} / k_{i}\right) / \partial k_{r}=0$ and $\partial\left(\omega_{i} / k_{i}\right) / \partial k_{i}=0$. Straightforward differentiation shows that the above equations are equivalent to $\partial \omega_{i} /\left.\partial k_{r}\right|_{k=k^{*}}=0$ and $v_{-}^{*}=\omega_{i}^{*} / k_{i}^{*}=\partial \omega_{i} /\left.\partial k_{i}\right|_{k=k^{*}}$, respectively. The criterion proposed by van Saarloos (1987) establishes that the $\mathrm{C} / \mathrm{A}$ instability transition occurs when $v_{-}^{*}=0$. Therefore, the system exhibits $\mathrm{C} / \mathrm{A}$ instability transition at the point in the parameter space where the dispersion relation has a solution $\left(\omega^{*}, k^{*}\right)$ for which

$$
\left.\frac{\partial \omega_{i}}{\partial k_{r}}\right|_{k=k^{*}}=\left.\frac{\partial \omega_{i}}{\partial k_{i}}\right|_{k=k^{*}}=\omega_{i}^{*}=0 .
$$

Assuming that $\omega=\omega(k)$ is an analytic function, the Cauchy-Riemann equations establish that $\partial \omega_{r} / \partial k_{r}=\partial \omega_{i} / \partial k_{i}$ and $\partial \omega_{r} / \partial k_{i}=-\partial \omega_{i} / \partial k_{r}$. From these equations and (A 1), one obtains $\mathrm{d} \omega /\left.\mathrm{d} k\right|_{k=k^{*}}=\omega_{i}^{*}=0$, which coincides with the classical saddle-point criterion (Briggs 1964; Huerre \& Monkewitz 1990).

\section{REFERENCES}

AnNA, S. L. \& MAYER H. C. 2006 Microscale tipstreaming in a microfluidic flow focusing device. Phys. Fluids. 18, 121512.

Basaran, O. A. 2002 Small-scale free surface flows with breakup: Drop formation and emerging applications. AIChE J. 48, 1842-1848.

BAUER, H. F. 1986 Free surface and interface oscillations of an infinitely long visco-elastic liquid column. Acta Astronautica 13, 9-22.

Brenn, G., LiU, Z. \& Durst, F. 2000 Linear analysis of the temporal instability of axisymmetrical non-Newtonian liquid jets. Intl J. Multiphase Flow 26, 1621-1644.

BRIGGS, R. J. 1964 Electron-Stream Interaction with Plasmas. MIT Press.

Cohen, I. \& Nagel, S. R. 2002 Scaling at the selective withdrawal transition through a tube suspended above the fluid surface. Phys. Rev. Lett. 88, 074501.

Coullet, P. \& Mahadevan, L. 2005 Hydrodynamical models for the chaotic dripping faucet. J. Fluid Mech. 526, 1-17.

Eggers, J. \& Villermaux, E. 2008 Physics of liquid jets. Rep. Prog. Phys. 71036601.

Eggleton, C. D., Tsai, T.-M. \& Stebe, K. J. 2001 Tip streaming from a drop in the presence of surfactants. Phys. Rev. Lett. 87, 048302.

FunAda, T. \& JoSEPH, D. D. 2003 Viscoelastic potential flow analysis of capillary instability. J. Non-Newtonian Fluid Mech. 111, 87-105.

GaÑán-CALVO, A. M. 1998 Generation of steady liquid microthreads and micron-sized monodisperse sprays in gas streams. Phys. Rev. Lett. 80, 285-288.

Gañán-Calvo, A. M., González-Prieto, R., Riesco-Chueca, P., Herrada, M. A. \& FloresMosquera, M. 2007 Focusing capillary jets close to the continuum limit. Nature Physics 3, 737-742.

Guillot, P., Colin, A., Utada, A. S. \& Ajdari, A. 2007 Stability of a jet in confined pressure-driven biphasic flows at low Reynolds numbers. Phys. Rev. Lett. 99, 104502. 
Huerre, P. \& Monkewitz, P. A. 1990 Local and global instabilities in spatially developing flows. Annu. Rev. Fluid Mech. 22, 473-537.

Joseph, D. D. 1990 Fluid Dynamics of Viscoelastic Flows. Springer.

Leib, S. J. \& GoldsteIn, M. E. 1986 The generation of capillary instabilities on a liquid jet. J. Fluid Mech. 168, 479-500.

Lin, S. P. 2003 Breakup of Liquid Sheets and Jets. Cambridge University Press.

LiU, Z. \& LiU, Z. 2006 Linear analysis of three-dimensional instability of non-Newtonian liquid jets. J. Fluid Mech. 559, 451-459.

Montanero, J. M. \& Gañán-Calvo, A. M. 2008 Stability of coflowing capillary jets under nonaxisymmetric perturbations. Phys. Rev. E 77, 046301.

de la Mora, J. F. 2007 The fluid dynamics of Taylor cones. Annu. Rev. Fluid Mech. 39, 217-243.

VAN SAARloos, W. 1987 Dynamical velocity selection: Marginal stability. Phys. Rev. Lett. 58, 25712574.

VAn SaArloos, W. 2003 Front propagation into unstable states. Phys. Rep. 386, 29-222.

Schroll, R. D., Wunenburger, R., Casner, A., Zhang, W. W. \& Delville, J.-P. 2007 Liquid transport due to light scattering. Phys. Rev. Lett. 98, 133601.

Stone, H. A., Stroock, A. D. \& Adjari, A. 2004 Engineering flows in small devices: Microfluidics toward a lab-on-a-chip. Annu. Rev. Fluid Mech. 36, 381-411.

Suryo, R. \& Basaran, O. A. 2006 Tip streaming from a liquid drop forming from a tube in a co-flowing outer fluid. Phys. Fluids 18, 082102.

TAYLOR, G. I. 1934 The formation of emulsions in definable fields of flow. Proc. R. Soc. Lond. A 146, 501-523.

Zhou, C., Yue, P. \& Feng, J. J. 2006 Formation of simple and compound drops in microfluidic devices. Phys. Fluids 18, 092105. 